Draft version March 19, 2018

Preprint typeset using $\mathrm{L}^{A} \mathrm{~T}_{\mathrm{E}} \mathrm{X}$ style emulateapj v. 01/23/15

\title{
REPEATED TRANSIENT JETS FROM A WARPED DISK IN THE SYMBIOTIC PROTOTYPE Z AND: A LINK TO THE LONG-LASTING ACTIVE PHASE
}

\author{
Augustin SKOPAL ${ }^{1}$ \\ Astronomical Institute, Slovak Academy of Sciences, 05960 Tatranská Lomnica, The Slovak Republic \\ TAYA. N. TARASOVA \\ Scientific Research Institute, Crimean Astrophysical Observatory, 298409 Nauchny, Crimea \\ MAREK WOLF \\ Astronomical Institute, Charles University Prague, CZ-180 00 Praha 8, V Holešovičkách 2, The Czech Republic \\ AND \\ Pavol A. DubovskÝ and Igor KudzeJ \\ Vihorlat Astronomical Observatory, Mierová 4, SK-066 01 Humenné, The Slovak Republic \\ Draft version March 19, 2018
}

\begin{abstract}
Active phases of some symbiotic binaries survive for a long time from years to decades. The accretion process onto a white dwarf (WD) sustaining long-lasting activity, and sometimes leading to collimated ejection, is not well understood. We present the repeated emergence of highly collimated outflows (jets) from the symbiotic prototype Z And during its 2008 and 2009-10 outbursts and suggest their link to the current long-lasting (from 2000) active phase. We monitored Z And with the high-resolution spectroscopy, multicolor $U B V R_{\mathrm{C}^{-}}$and high-time-resolution-photometry. The well-pronounced bipolar jets were ejected again during the 2009-10 outburst together with the simultaneous emergence of the rapid photometric variability $(\Delta m \approx 0.06 \mathrm{mag}$ ) on the timescale of hours, showing similar properties as those during the 2006 outburst. These phenomena and the measured disk-jets connection could be caused by the radiation-induced warping of the inner disk due to a significant increase of the burning WD luminosity. Ejection of transient jets by Z And around outburst maxima signals a transient accretion at rates above the upper limit of the stable hydrogen burning on the WD surface. The enhanced accretion through the disk warping, supplemented by the accretion from the giant's wind, can keep a high luminosity of the WD for a long time, until depletion of the disk. In this way, the jets provide a link to long-lasting active phases of $\mathrm{Z}$ And.
\end{abstract}

Subject headings: Stars: binaries: symbiotic - stars: individual: (Z And) - ISM: jets and outflows

\section{INTRODUCTION}

Symbiotic stars are the widest interacting binaries with orbital periods of a few years. They consist of a red giant (RG) as the donor and a white dwarf (WD) as the accretor (Boyarchuk 1967; Kenyon 1986). Symbiotic stars are well detached binary systems (Mürset \& Schmid 1999), which implies that their activity is triggered via the wind mass transfer.

According to the behavior of optical light curves (LC) we distinguish between the quiescent and active phases. During quiescent phases, the WD accretes throughout the accretion disk formed from the giant's wind. This process heats up the WD to > $10^{5} \mathrm{~K}$ and increases its luminosity to $\sim 10^{1}-10^{4} L_{\odot}$, which in return ionizes a portion of the neutral wind from the giant, giving rise to the nebular emission (Seaquist et al. 1984). Optical LCs are characterized with a periodic wave-like variation. No sudden brightenings are indicated. On the other hand, active phases are characterized by a few magnitude (multiple) brightening(s)-outbursts-observed on the timescale of a few months to years/decades (see, e.g., historical
LCs of FN Sgr, Z And, AX Per of Brandi et al. 2005; Leibowitz \& Formiggini 2008, 2013) with signatures of a mass outflow (e.g., Fernández-Castro et al. 1995; Esipov et al. 2000; Sokoloski et al. 2006; McKeever et al. 2011). They are called ' $\mathrm{Z}$ And-type' outbursts, because they were observed in the past for a prototype of the class of symbiotic stars - Z And Kenyon 1986). In rare cases, these outbursts are followed by collimated ejection. To date, its signature in the optical spectra has been recorded only for a few objects: MWC 560 (e.g. Tomov et al. 1990), Hen 31341 (Tomov et al. 2000; Munari et al. 2005), StH $\alpha 190$ (Munari et al. 2001), Z And (Skopal \& Pribulla 2006), BF Cyg (Skopal et al. 2013) and St 2-22 (Tomov et al. 2017).

The Z And binary consists of a late-type M4.5 III RG and a WD accreting from the giant's wind on the 758-day orbit (e.g. Nussbaumer \& Vogel 1989; Fekel et al. 2000). During the quiescent phase, a high luminosity of its hot component, on the order of $10^{3} L_{\odot}$ (Mürset et al. 1991; Skopal 2005), is generated by the stable nuclear burning of hydrogen on the WD surface (e.g., Paczyński \& Rudak

${ }^{1}$ E-mail: skopal@ta3.sk 

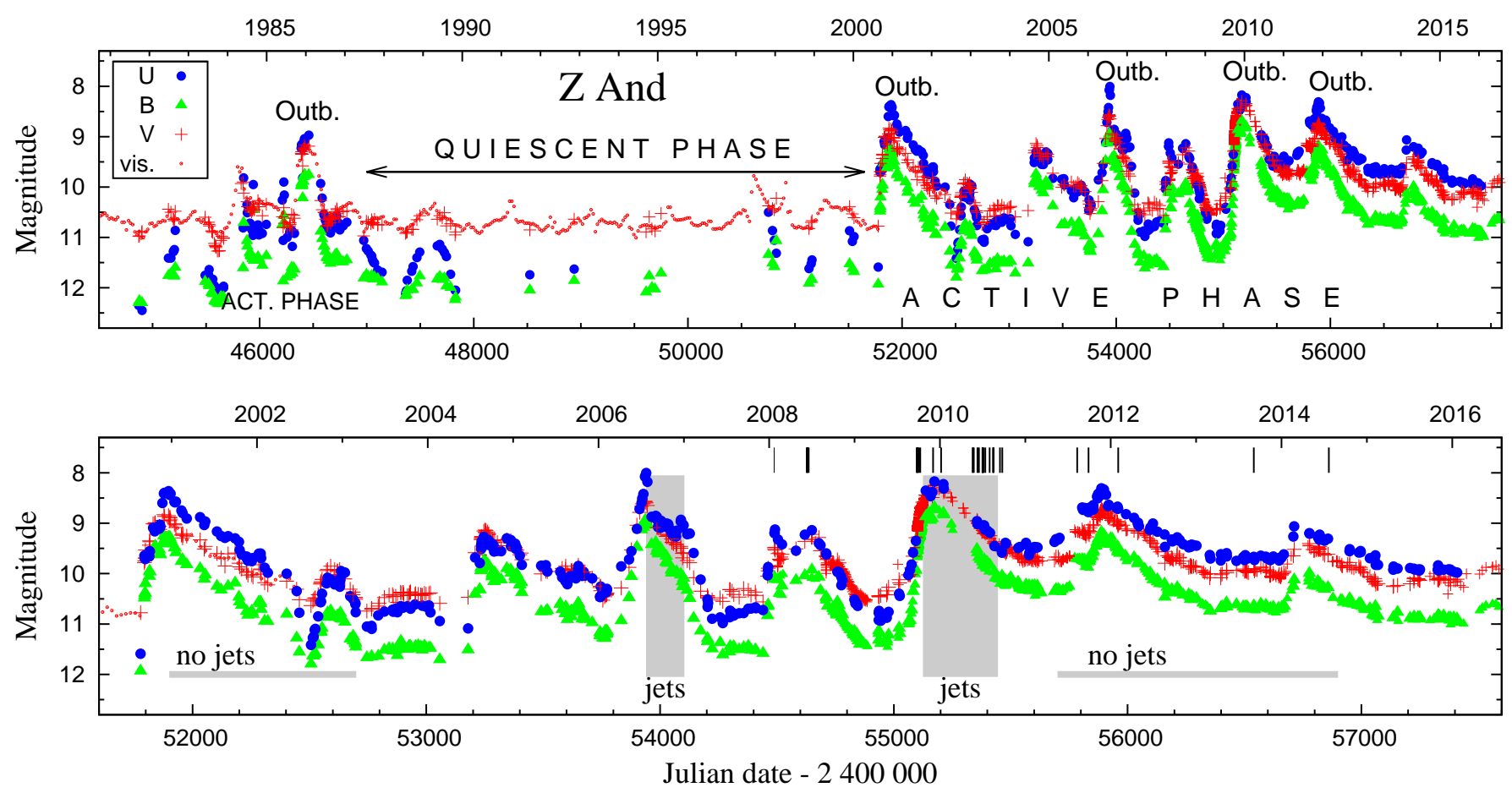

FIG. 1.- Top: $U B V$ light curves of $\mathrm{Z}$ And from 1981 covering its quiescent and active phases. References for the data used are in Skopal et al. (2000). Bottom: Current active phase that started in September 2000 showing multiple outbursts. Vertical bars indicate times of our spectra (Table 1). The presence of bipolar jets is denoted by vertical gray belts. No jets were indicated during the major 2000-03 outburst (Skopal et al. 2006) and during 2011-12 and 2014 brightenings (Fig. 3). Data are described in Sect. 2.2.

1980). We call these systems nuclearly powered 2 In September 2000, Z And started a new active phase showing a series of outbursts with the main optical maxima in December 2000, July 2006, December 2009 and December 2011 (see Fig. 1). At/after the 2006 maximum, highly collimated bipolar jets were detected for the first time as the satellite emission components to $\mathrm{H} \alpha$ and $\mathrm{H} \beta$ lines (Skopal \& Pribulla 2006). Their presence was confirmed by Burmeister \& Leediärv (2007) and Tomov et al. (2007). Temporal development of jets and their possible origin were investigated by Skopal et al. (2009). The event of jets was transient, being detected through the end of 2006, along the decrease of the star's brightness. During this period, a low amplitude irregular photometric variation from the low stage $(\lesssim 0.02 \mathrm{mag})$ increased its amplitude to $\sim 0.06 \mathrm{mag}$ on the timescale of hours. Its source was identified with a large disk around the WD. The authors ascribed these phenomena to a disruption of the inner parts of the disk caused by its radiation-induced warping.

In this contribution, we present an analysis of collimated mass outflow, which we measured again during the 2008 and 2009-10 outbursts. We compare the new jet event with that from the 2006 outburst to establish a more trustworthy disk-jets connection. In this way, we aim to understand better accretion process onto the WD that keeps the current active phase of $\mathrm{Z}$ And for a long time, showing outbursts with or without jets. Our observations are described in Sect. 2, Their analysis and results are introduced in Sect. 3. A discussion of the

\footnotetext{
${ }^{2}$ For a comparison, accretion-powered systems generate the luminosity of the order of $10^{1} L \odot$ by converting the gravitational potential energy of the WD via the accretion disk.
}

results and a summary are provided in Sects. 4 and 5 . respectively.

\section{OBSERVATIONS AND DATA REDUCTION}

Our observations of $\mathrm{Z}$ And during its current active phase, from 2008 to 2014, were carried out at different observatories.

\subsection{Spectroscopy}

(i) At the Crimean Astrophysical Observatory (denoted as CrAO in Table 1) the high-resolution spectra were performed with the coudé spectrograph of the 2.6 $m$ ZTSH telescope. The size of the ANDOR IKONL CCD detector DZ936N was $2048 \times 2048$ pixels. The spectral resolving power was $\mathrm{R} \sim 25000$ around $\mathrm{H} \alpha$. The low-resolution spectra $(\mathrm{R} \sim 1000)$ were obtained using a spectrograph mounted at the Nasmyth telescope focus. The detector was a SPEC-10 CCD camera $(1340 \times 100$ pixels). The spectrophotometric standard HR 8965 was observed each night to calibrate the measured flux in the star's spectrum. The primary reduction of the spectra, including the bias subtraction and flat fielding, was performed with the SPERED code developed by S. I. Sergeev at the $\mathrm{CrAO}$.

(ii) At the Ondřejov Observatory (Ondr.), spectra of $\mathrm{Z}$ And were secured with a SITe-005 $800 \times 2030$ CCD detector attached to the medium 0.7-m camera of the coudé focus of the Ondřejov $2.0 \mathrm{~m}$ telescope. The spectra were obtained between June 2010 and July 2014, and have a linear dispersion of $17.2 \AA / \mathrm{mm}$ and a 2-pixel resolving power of about $12600\left(11-12 \mathrm{kms}^{-1}\right.$ per pixel). Standard initial reduction of CCD spectra was carried out using modified MIDAS and IRAF packages. Final pro- 
TABLE 1

LOG OF SPECTROSCOPIC OBSERVATIONS

\begin{tabular}{|c|c|c|c|}
\hline $\begin{array}{c}\text { Date } \\
(\mathrm{mm} / \mathrm{dd} / \text { yyyy })\end{array}$ & $\begin{array}{r}\text { JD } \\
245 . .\end{array}$ & $\begin{array}{c}\text { Region } \\
\text { (nm) }\end{array}$ & Obs. $^{\star}$ \\
\hline \multicolumn{4}{|c|}{2008 burst } \\
\hline $01 / 23 / 2008$ & 4488.547 & $642-670$ & $\mathrm{DDO}$ \\
\hline $06 / 11 / 2008$ & 4628.844 & $642-670$ & $\mathrm{DDO}$ \\
\hline $06 / 11 / 2008$ & 4628.856 & $461-491$ & $\mathrm{DDO}$ \\
\hline $06 / 13 / 2008$ & 4630.836 & $642-670$ & $\mathrm{DDO}$ \\
\hline $06 / 19 / 2008$ & 4636.822 & $642-670$ & DDO \\
\hline \multicolumn{4}{|c|}{ 2009-10 outburst } \\
\hline $09 / 26 / 2009$ & 5100.535 & $335-740$ & $\mathrm{CrAO}^{a}$ \\
\hline $10 / 01 / 2009$ & 5106.237 & $653-659$ & $\mathrm{CrAO}^{b}$ \\
\hline $10 / 08 / 2009$ & 5113.297 & $653-659$ & $\mathrm{CrAO}^{b}$ \\
\hline $12 / 02 / 2009$ & 5168.235 & $653-659$ & $\mathrm{CrAO}^{b}$ \\
\hline $01 / 05 / 2010$ & 5202.273 & $651-661$ & $\mathrm{CrAO}^{b}$ \\
\hline $05 / 21 / 2010$ & 5337.499 & $353-757$ & $\mathrm{CrAO}^{a}$ \\
\hline $05 / 25 / 2010$ & 5342.465 & $653-661$ & $\mathrm{CrAO}^{b}$ \\
\hline $06 / 10 / 2010$ & 5358.508 & $626-677$ & Ondr. \\
\hline $06 / 16 / 2010$ & 5364.561 & $626-677$ & Ondr. \\
\hline $07 / 01 / 2010$ & 5379.472 & $651-661$ & $\mathrm{CrAO}^{b}$ \\
\hline $07 / 02 / 2010$ & 5380.503 & $622-674$ & Ondr. \\
\hline $07 / 13 / 2010$ & 5391.509 & $626-677$ & Ondr. \\
\hline $08 / 01 / 2010$ & 5410.481 & $651-661$ & $\mathrm{CrAO}^{b}$ \\
\hline $09 / 24 / 2010$ & 5464.434 & $647-665$ & $\mathrm{CrAO}^{b}$ \\
\hline \multicolumn{4}{|c|}{2012 and 2014 brightenings } \\
\hline $08 / 10 / 2011$ & 5784.379 & $639-691$ & Ondr. \\
\hline $09 / 28 / 2011$ & 5833.270 & $640-691$ & Ondr. \\
\hline $02 / 02 / 2012$ & 5960.221 & $640-691$ & Ondr. \\
\hline $09 / 04 / 2013$ & 6540.445 & $641-688$ & Ondr. \\
\hline $07 / 22 / 2014$ & 6861.495 & $641-688$ & Ondr. \\
\hline
\end{tabular}

Notes: ${ }^{\star}$ Observatory, ${ }^{a}$ Nasmyth, ${ }^{b}$ coudé

cessing of the data was done with the aid of the SPEFOpackage software (Horn et al. 1996; Skoda 1996).

(iii) At the David Dunlap Observatory, University of Toronto (DDO), spectra of Z And were performed with a Jobin Yovon Horiba CCD detector $(2048 \times 512$ pixels of $13.5 \mu \mathrm{m}$ size) attached to the single dispersion slit spectrograph of the Cassegrain focus of the $1.88 \mathrm{~m}$ telescope. The slit width was $240 \mu \mathrm{m}$ corresponding to 1.5 arcsec at the focal plane. Around the $\mathrm{H} \alpha$ region, the resolving power was $\sim 12000$. Standard reduction of the spectra was performed with the IRAF-package software.

A journal of the spectra is given in Table 1.

\subsection{Photometry}

(iv) At the Skalnaté Pleso and Stará Lesná (pavilion G2) observatories, classical photoelectric $U B V R_{\mathrm{C}}$ measurements were carried out by single-channel photometers mounted in the Cassegrain foci of $0.6 \mathrm{~m}$ reflectors. Data are plotted in Fig. 1. Each value represents the average of the observations during a night. Corresponding inner uncertainties are of a few times $0.01 \mathrm{mag}$ in the $B, V$ and $R_{\mathrm{C}}$ bands, and up to $0.05 \mathrm{mag}$ in the $U$ band (see Vaňko et al. 2015a,b). The data were published by Skopal et al. (2012). New observations (from November 2011) will be published elsewhere (Sekeráš et al., in preparation). To get a better coverage of the investigated period, we complemented our photometry with the $B V R_{\mathrm{C}} \mathrm{CCD}$ measurements available at the AAVSO database. 3 .

(v) At the Stará Lesná observatory (pavilion G1) the high-time resolution CCD photometry was per-

\footnotetext{
${ }^{3}$ https://www.aavso.org/data-download
}

formed during nights on 06/24/2007, 09/21/2007 and $07 / 11 / 2008$. The SBIG ST10 MXE CCD camera $(2184 \times 1472$ pixels; $6.8 \mu \mathrm{m})$ mounted at the Newtonian focus of the $0.5 \mathrm{~m}$ telescope was used (see Parimucha \& Vaňko 2005, in detail). The star $\mathrm{BD}+474192(V=8.99, B-V=0.41, U-B=0.14$, $V-R_{\mathrm{C}}=0.19$; Skopal et al. 2012, and references therein) was used as the standard star for both photoelectric and CCD observations.

(vi) At the Astronomical Observatory on the Kolonica Saddle, the fast CCD photometry was performed during the nights on 11/01/2009, 11/02/2009 and 08/14/2011. A FLI Pro Line PL1001E CCD camera with the chip $1024 \times 1024$ pixels $(24 \mu \mathrm{m})$ was attached to the Ritchey-Chretien telescope $300 / 2400 \mathrm{~mm}$. The star TYC3641-00678-1 $(\mathrm{B}=9.43, \mathrm{~V}=9.05, \mathrm{Rc}=8.83, \mathrm{Ic}=8.62$, Henden \& Honevcutt 1997) was used as the comparison. All CCD frames were dark subtracted, flat-fielded, and corrected for cosmic rays. Our high-time-resolution photometry is plotted in Fig. 4 .

Arbitrary flux units of the high-resolution spectra around the $\mathrm{H} \alpha$ line were converted to absolute fluxes with the aid of $R_{\mathrm{C}}$ magnitudes corrected for the $\mathrm{H} \alpha$ equivalent width (see Eq. (10) of Skopal 2007). Magnitudes were converted to fluxes according to the calibration of Henden \& Kaitchuck (1982) and Bessel (1979). Observations were dereddened with $E_{\mathrm{B}-\mathrm{V}}=0.30$ and resulting parameters were scaled to a distance of $1.5 \mathrm{kpc}$ (Mürset et al. 1991; Mikołajewska \& Kenyon 1996). Orbital phase was calculated according to the ephemeris of the inferior conjunction of the cool giant (Fekel et al. 2000) as

$$
J D_{\text {spec.conj. }}=2450260.2+759.0 \times E .
$$

\section{ANALYSIS AND RESULTS}

\subsection{Photometric evolution}

Figure 1 shows the $U B V$ LCs of $\mathrm{Z}$ And covering its quiescent and active phases from 1981. The recent active phase began in September 2000 (Skopal et al. 2000). Our spectra (Table 1) cover three brightenings in the recent evolution of the LC. The burst in 2008 with $U_{\max } \sim 9.2$, the main outburst that peaked at $U \sim 8.2$ on December 2009 and the 2012 and 2014 brightenings with $U_{\max } \sim 8.4$ and $\sim 9.0$.

To justify the connection between the rapid photometric variability and ejection of jets as suggested by Skopal et al. (2009), we searched for a short-term photometric variability on the timescales of minutes to hours. During the 2007 quiescent phase, prior to the 2008 burst, we observed an irregular variation within $\Delta B \lesssim 0.02 \mathrm{mag}$, comparable with that of standard stars. During the 2008 burst, we observed light variations with $\Delta B \sim 0.025 \mathrm{mag}$ on the timescale of $1-2$ hours, whereas at the maximum of the main 2009 outburst, the light variations increased to $\Delta B \sim 0.065 \mathrm{mag}$ and enlarged its timescale to 7-9 hours throughout the whole night (Fig. (4). Similar evolution in the rapid photometric variability was also indicated during the previous 2006 major outburst, when the jet features were measured for the first time (see Fig. 3 of Skopal et al. 2009).

\subsection{Evolution of the $H \alpha$ profile}



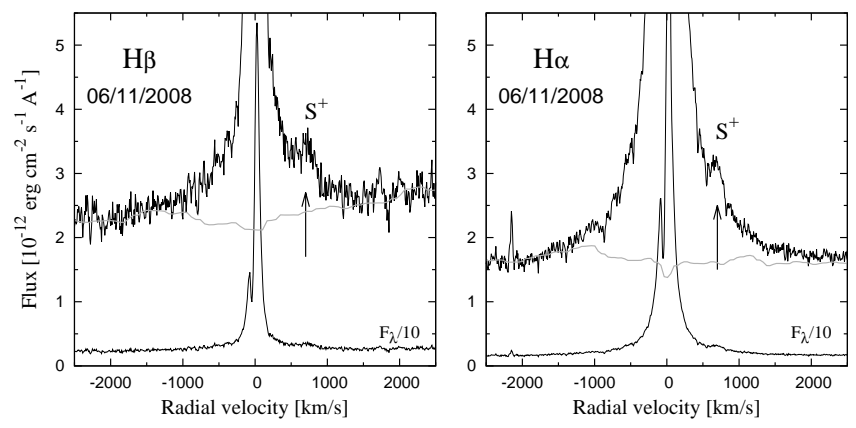

FIG. 2. Example of the $\mathrm{H} \beta$ and $\mathrm{H} \alpha$ profile observed at the maximum of the 2008 burst. Only the $\mathrm{S}^{+}$satellite component was indicated at $\sim 700 \mathrm{~km} \mathrm{~s}^{-1}$. The gray line represents the model continuum of a normal M5 giant according to Fluks et al. (1994).

During the 2008 burst, the $\mathrm{H} \alpha$ line showed broad wings extended to $\sim \pm 1500 \mathrm{~km} \mathrm{~s}^{-1}$ and a sharp absorption cutting the emission core at $\sim-50 \mathrm{~km} \mathrm{~s}^{-1}$. A faint $S^{+}$satellite component was seen at $\sim 700 \mathrm{kms}^{-1}$ (Fig. 3, spectra from January 23 to June 19, 2008). Its presence was confirmed by a similar feature in the $\mathrm{H} \beta$ profile (Fig. 2).

During the major 2009-10 outburst, the $\mathrm{H} \alpha$ profile was also of a P-Cyg type with broad wings (Fig. 3, spectra from September 26, 2009 to September 24, 2010). The first satellite components were recorded on September 26 , 2009 , prior to the light maximum, at $\sim \pm 1100 \mathrm{~km} \mathrm{~s}^{-1}$. However, at the maximum of the star's brightness (our spectrum from $12 / 02 / 2009)$ the satellite components were hardly recognizable. They appeared again when the brightness of $\mathrm{Z}$ And began to fall (spectrum from January 5, 2010). Their position with respect to the reference wavelength increased to $\pm(1700-1800) \mathrm{kms}^{-1}$ during January to September 2010 (Table 2, Fig. 6), when the star's brightness gradually decreased by $\sim 1$ magnitude in $U$, from $\sim 8.2$ to $\sim 9.2$ (Fig. 1).

The last of our spectra taken around the following brightenings (December 2011 and January 2014; see Fig. 1) did not show any clear signatures of satellite components in the $\mathrm{H} \alpha$ profile. We observed a simple emission core with a reduced wings and the width of the line with respect to the 2009-10 outburst (Fig. 3).

\subsection{Parameters of satellite components}

To determine measured parameters of the satellite components, we isolated them from the whole line profile by fitting the emission line core and its extended wings with two Gaussian functions. Then the residual satellite emissions were compared with additional Gaussians. Using their fitted parameters (the central wavelength, maximum, $I$, and the width $\sigma$ ) we determined the radial velocity of satellite components, $R V_{\mathrm{S}}$, their flux $F_{\mathrm{S}}=\sqrt{2 \pi} I \sigma$ and the width $F W H M_{\mathrm{S}}=2 \sqrt{2 \ln (2)} \sigma$. Resolution of our spectra allowed us to estimate uncertainties in $R V_{\mathrm{S}}$ to $10-25 \mathrm{~km} \mathrm{~s}^{-1}$, in $F_{\mathrm{S}}$ fluxes within $10-20 \%$ of the observed values and in $F W H M_{\mathrm{S}}$ widths within $0.2-0.3 \AA$. The results are given in Table 2 .

\subsection{Physical parameters of jets}

According to reasons discussed by Skopal et al. (2009), the geometry of the emitting medium that gives rise to the satellite components can be approximated by a narrow cone with the peak at the central object character- ized with an opening angle $\theta_{0}$. This implies that the measured narrow satellite components can be produced by highly collimated emitting particles - jets. Therefore, using the measured parameters of the satellite components, we can determine some physical parameters of jets.

\subsubsection{Opening angle}

Assuming that jets were launched with a constant velocity, $v_{\text {jet }}$, perpendicularly to the disk plane, which coincides with the orbital one (i.e. $R V_{\mathrm{S}}=v_{\text {jet }} \cos (i)$ ), the opening angle can be approximated as

$$
\theta_{0}=2 \sin ^{-1}\left[\frac{H W Z I_{\mathrm{S}}}{R V_{\mathrm{S}} \tan (i)}\right],
$$

where $i$ is the orbital inclination (see Skopal et al. 2009). Corresponding parameters from Table 2. orbital inclination $i=63 .^{\circ} 6+6 .^{\circ} 9 /-12 .^{\circ} 2$ (Shagatova 2017) and adopting $H W Z I_{\mathrm{S}}=F W H M_{\mathrm{S}}$ yield the average value of the jet opening angle as

$\theta_{0}=16 .^{\circ} 5+4 .^{\circ} 7 /-8 .^{\circ} 3$ for 2008 burst

and

$\theta_{0}=9 .^{\circ} 4+2 .^{\circ} 8 /-4 .^{\circ} 8$ for $2009-10$ outburst,

after its maximum. Prior to the maximum of the 200910 outburst, $\theta_{0} \sim 15 .^{\circ} 2$. Uncertainties were determined as standard error of function (2) using its total differential for the uncertainty in $i$. Influence of uncertainties in $F W H M_{\mathrm{S}}$ and $R V_{\mathrm{S}}$ (Sect. 3.3) can be neglected. Individual values of $\theta_{0}$ are given in Table2. Figure 6 depicts also data from the 2006 outburst recalculated for $i=63 .^{\circ} 6$.

\subsubsection{Emission measure}

Assuming that (i) the jet radiation is produced by the recombination transitions in the $\mathrm{H} \alpha$ line, (ii) the medium is completely ionized, (i.e. $n_{\mathrm{e}}=n_{\mathrm{p}} \equiv \bar{n}_{\text {jet }}$ ) and radiates at a constant electron temperature $T_{\mathrm{e}}$, the luminosity of jets in $\mathrm{H} \alpha$ is related to the line emissivity, $\varepsilon_{\alpha} \bar{n}_{\text {jet }}^{2}$, by

$$
L_{\text {jet }}(\mathrm{H} \alpha)=\varepsilon_{\alpha} \bar{n}_{\text {jet }}^{2} V_{\text {jet }},
$$

where $\varepsilon_{\alpha}$ is the volume emission coefficient in $\mathrm{H} \alpha, \bar{n}_{\text {jet }}$ is the mean particle concentration and $V_{\text {jet }}$ is the volume of the jets. For the optically thin medium of jets, the luminosity can be determined from the observed fluxes as $L_{\text {jet }}=4 \pi d^{2} \times F_{\mathrm{S}}$. According to the definition of the emission measure, $E M_{\text {jet }}=\bar{n}_{\text {jet }}^{2} V_{\text {jet }}$, and assumptions above, we can express it as,

$$
E M_{\text {jet }}(\mathrm{H} \alpha)=4 \pi d^{2} \frac{F_{\mathrm{S}}}{\varepsilon_{\alpha}} .
$$

The emission measure for one jet is of a few $\times 10^{57} \mathrm{~cm}^{-3}$ for $\varepsilon_{\alpha}\left(T_{\mathrm{e}}=2 \times 10^{4} \mathrm{~K}\right)=1.83 \times 10^{-25} \mathrm{erg} \mathrm{cm}^{3} \mathrm{~s}^{-1}$ (e.g. Osterbrock 1989) (Table 2).

\subsubsection{Radius}

The conical shape of jets defines their volume as $V_{\text {jet }}=$ $1 / 3 R_{\text {jet }}^{3} \times \Delta \Omega$, where $\Delta \Omega=2 \pi\left[1-\cos \left(\theta_{0} / 2\right)\right]$ is the solid angle of the jet nozzle. Using Eq. (4) the jet radius, $R_{\text {jet }}$, can be expressed by means of the parameters $L_{\text {jet }}$ and 

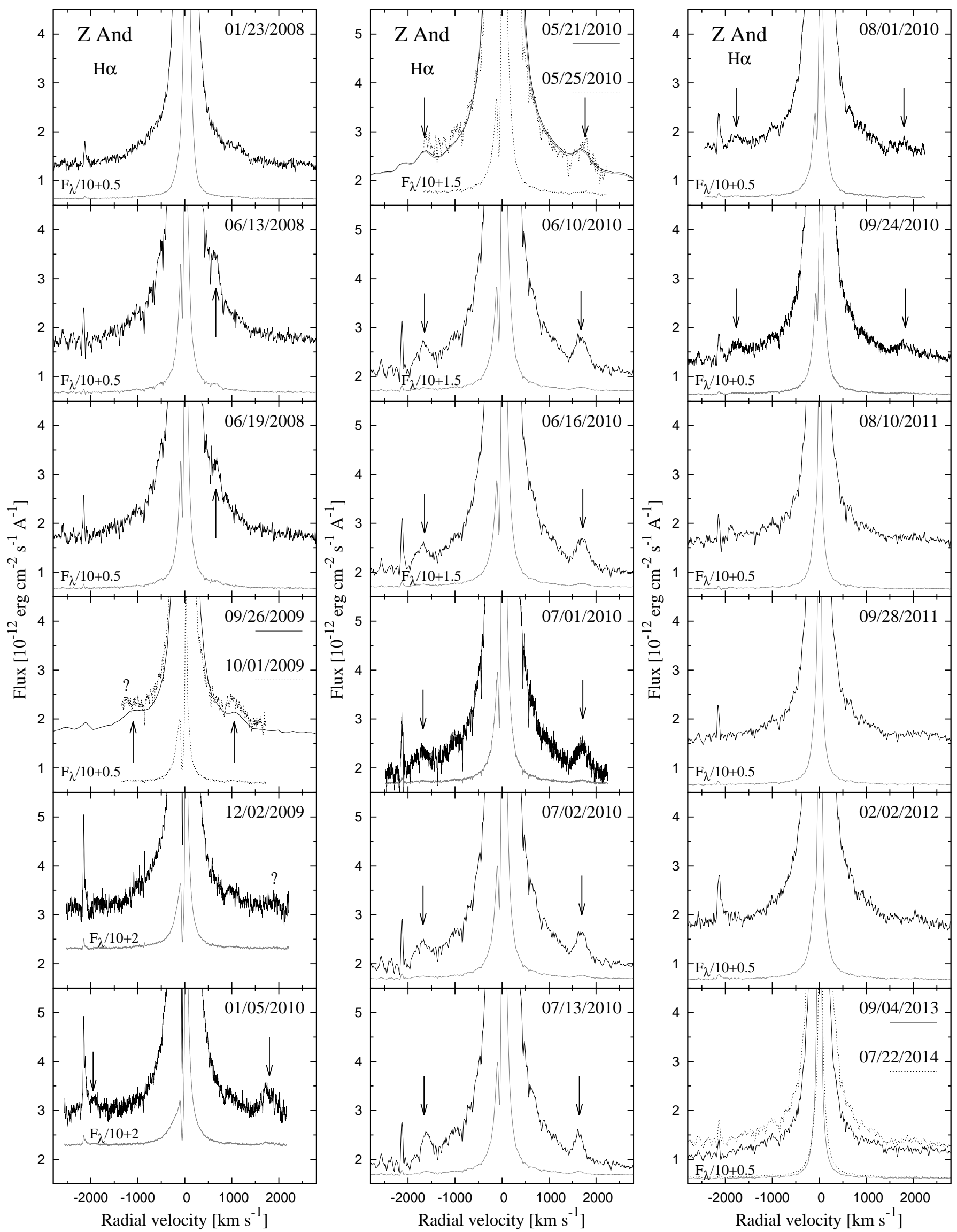

FIG. 3.- Evolution of the $\mathrm{H} \alpha$ broad wings in our spectra (Sect. 3.2). The satellite components are denoted by arrows. Their parameters are in Table 2 When the coverage of broad wings was insufficient, we compared low-resolution spectra (09/26/2009 and 05/21/2010). The presence or absence of bipolar jets along the current active phase is denoted in Fig. 1 

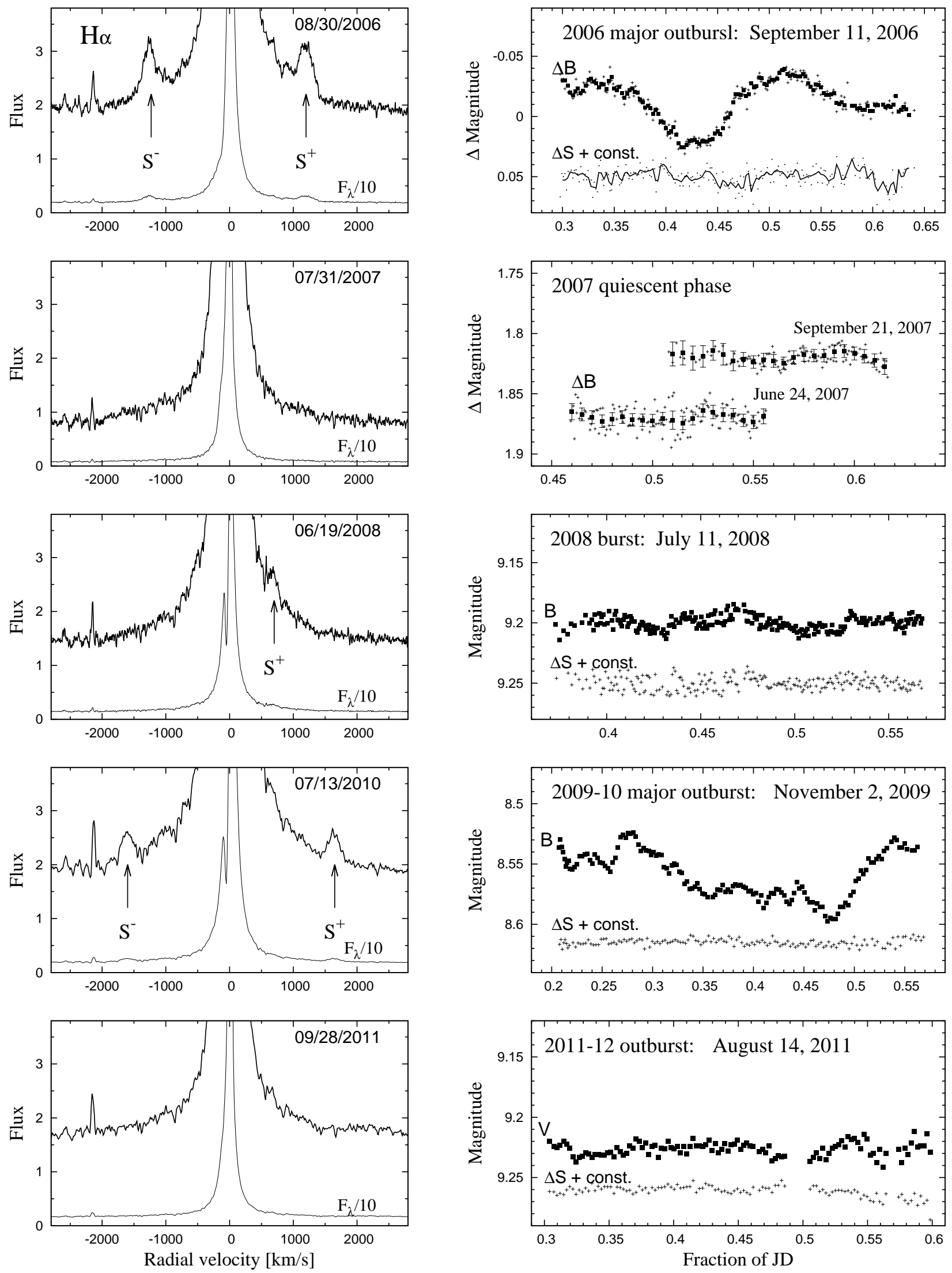

FIG. 4.- H $\alpha$ broad wings (left panels) and short-term variability in the optical continuum (right panels) at different stages of $\mathrm{Z}$ And activity. During the 2006 and 2009-10 major outbursts, when the satellite $\mathrm{S}^{-}$and $\mathrm{S}^{+}$components emerged, a slow higher-amplitude photometric variation developed (Sect. 3.1). Data from 2006 and 2007 were published by [Skopal et al. (2009). Fluxes are in $10^{-12} \mathrm{erg} \mathrm{cm}^{-2} \mathrm{~s}^{-1} \AA^{-1}$. 
TABLE 2

Parameters of Gaussian fits to the Satellite emission COMPOnEnts $S^{-}$and $S^{+}$: Radial Velocity $R V_{\mathrm{S}}\left(\mathrm{Km} \mathrm{S} \mathrm{S}^{-1}\right)$, Flux $F_{\mathrm{S}}$

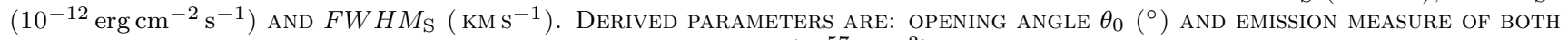
JETS, $\mathrm{EM}_{\text {jet }}\left(10^{57} \mathrm{CM}^{-3}\right)$

\begin{tabular}{cccccccccc}
\hline Date & \multicolumn{2}{c}{$R V_{\mathrm{S}}$} & \multicolumn{3}{c}{$F_{\mathrm{S}}$} & \multicolumn{3}{c}{$F W M_{\mathrm{S}}$} & \multicolumn{2}{c}{$\theta_{0}$} & $\mathrm{EM}_{\text {jet }}$ \\
\hline $\mathrm{mm} / \mathrm{dd} /$ yyyy & $\mathrm{S}^{-}$ & $\mathrm{S}^{+}$ & $\mathrm{S}^{-}$ & $\mathrm{S}^{+}$ & $\mathrm{S}^{-}$ & $\mathrm{S}^{+}$ & $\mathrm{S}^{-}$ & $\mathrm{S}^{+}$ & \\
\hline $06 / 11 / 2008$ & - & 707 & - & 2.3 & - & 194 & - & 15.7 & 3.4 \\
$06 / 13 / 2008$ & - & 661 & - & 3.0 & - & 215 & - & 18.6 & 4.4 \\
$06 / 19 / 2008$ & - & 693 & - & 1.7 & - & 183 & - & 15.1 & 2.5 \\
$09 / 26 / 2009^{\star}$ & -1157 & 1105 & & & & & & & \\
$10 / 01 / 2009^{\dagger}$ & & 1060 & & 2.3 & & 290 & & 15.6 & \\
$10 / 08 / 2009^{\dagger}$ & & 1040 & & 2.2 & & 269 & & 14.8 & \\
$12 / 02 / 2009$ & & & & & no & jets & & & \\
$01 / 05 / 2010$ & -2000 & 1790 & 3.3 & 5.1 & 301 & 301 & 8.57 & 9.58 & 12.4 \\
$05 / 21 / 2010^{\star}$ & -1670 & 1740 & & & & & & & \\
$05 / 25 / 2010^{\dagger}$ & & 1750 & & 5.4 & & 312 & & 10.1 & \\
$06 / 10 / 2010$ & -1714 & 1698 & 4.0 & 4.8 & 274 & 282 & 9.10 & 9.45 & 12.9 \\
$06 / 16 / 2010$ & -1723 & 1721 & 3.7 & 4.2 & 296 & 290 & 9.78 & 9.60 & 11.6 \\
$07 / 01 / 2010$ & -1723 & 1720 & 3.1 & 3.7 & 301 & 290 & 9.95 & 9.60 & 10.0 \\
$07 / 02 / 2010$ & -1723 & 1703 & 3.1 & 3.9 & 290 & 280 & 9.59 & 9.36 & 10.3 \\
$07 / 13 / 2010$ & -1625 & 1639 & 3.1 & 3.1 & 247 & 247 & 8.65 & 8.58 & 9.1 \\
$08 / 01 / 2010$ & -1750 & 1750 & 1.8 & 2.0 & 301 & 301 & 9.80 & 9.80 & 5.6 \\
$09 / 24 / 2010$ & -1755 & 1830 & 1.3 & 1.3 & 280 & 290 & 9.10 & 9.02 & 5.3 \\
\hline Notes: low-resolution & \multicolumn{7}{c}{ spectrum, ${ }^{\dagger}$ spectrum does not cover the $S^{-}$component }
\end{tabular}

$\Delta \Omega$ as a function of $\bar{n}_{\text {jet }}$,

$$
R_{\text {jet }}=\left(\frac{3 L_{\text {jet }}(\mathrm{H} \alpha)}{\varepsilon_{\alpha}\left(H, T_{\mathrm{e}}\right) \Delta \Omega} \bar{n}_{\text {jet }}^{-2}\right)^{1 / 3} .
$$

For average values of $F_{\mathrm{S}}$ and $\theta_{0}$ of one jet measured during 2010 (Table 2, Eq. (3)), the average jet radius can be expressed as a function of $\bar{n}_{\text {jet }}$ as

$$
\log \left(R_{\text {jet }} / \mathrm{AU}\right)=6.8-\frac{2}{3} \log \left(\bar{n}_{\text {jet }}\right) .
$$

\subsubsection{Mass-loss rate}

According to the mass continuity equation, the geometrical and kinematics parameters of the jets allow us to determine the mass-loss rate through jets as

$$
\dot{M}_{\text {jet }}=\Delta \Omega R_{\text {jet }}^{2} \mu m_{\mathrm{H}} \bar{n}_{\text {jet }} v_{\text {jet }},
$$

where $\mu$ is the mean molecular weight and $m_{\mathrm{H}}$ is the mass of the hydrogen atom. For the average value of the jet velocity during $2010, v_{\text {jet }}=R V_{\mathrm{S}} / \cos (i)=3900 \mathrm{~km} \mathrm{~s}^{-1}$ (Table 2) and the angle of the jet nozzle of $9 .^{\circ} 4$, we obtain

$$
\log \left(\dot{M}_{\text {jet }} / M_{\odot \mathrm{yr}^{-1}}\right)=-2.6-\frac{1}{3} \log \left(\bar{n}_{\text {jet }}\right)
$$

and/or as a function of the radius $R_{\text {jet }}$ as

$$
\log \left(\dot{M}_{\text {jet }} / M_{\odot \mathrm{yr}^{-1}}\right)=-6.0+\frac{1}{2} \log \left(R_{\text {jet }} / \mathrm{AU}\right)
$$

for one jet. Determination of $\dot{M}_{\text {iet }}$ thus requires a knowledge of $\bar{n}_{\text {jet }}$ or $R_{\text {jet }}$ (see Skopal et al. 2009, for details).

\subsection{Comparison with the 2006 outburst}

To date, bipolar jets in the form of satellite components to the $\mathrm{H} \alpha(\mathrm{H} \beta)$ line were identified in the spectrum of Z And only during the 2006 and 2009-10 outbursts (Fig. 1). Our monitoring of the latter showed that the spectroscopic and photometric characteristics of both outbursts are very similar. We summarize them as follows:

(i) The jets appeared around their optical maxima, and were gradually vanishing along the decrease of the star's brightness. Thus the event of jets was transient (Figs. 6 and 71).

(ii) In both cases, large variation in $R V_{\mathrm{S}}$ were observed at the beginning of their emergence, around optical maxima, and then settled on a constant level (see Fig. 2 of Tomov et al. (2007), Fig. 6 of Skopal et al. (2009) and Fig. 6 here).

(iii) During both outbursts, rapid light variation on the timescale of hours within $\Delta m \sim 0.06 \mathrm{mag}$ developed from irregular $\lesssim 0.02 \mathrm{mag}$ fluctuations outside the outbursts. (see Fig. 3 in Skopal et al. (2009) and Fig. 4 here).

The outbursts diversified only in duration of the star's brightness decline with jets, and in $R V_{\mathrm{S}}$. For the 2009-10 outburst, the period with jets lasted for $\approx 10$ months and satellite components settled on $\pm(1700-1800) \mathrm{km} \mathrm{s}^{-1}$, whereas for the 2006 outburst the relevant quantities were $\approx 5$ months and $\sim \pm 1100 \mathrm{~km} \mathrm{~s}^{-1}$, respectively (Figs. 6] and [7).

\section{DISCUSSION}

Here we discuss basic conditions of the jet's ejection by the nuclearly powered WD in the symbiotic binary $\mathrm{Z}$ And, and its possible consequences for the accretion process during $\mathrm{Z}$ And-type outbursts of symbiotic stars.

Skopal et al. (2009) interpreted the ejection of jets during the 2006 outburst as a result of disruption of the inner parts of the disk due to the radiation-induced warping caused by a significant increase of the WD luminosity at the outburst maximum. Close similarity of both the outbursts with jets (Sect. 3.5) suggests their common nature.

However, there is no theoretical application of the radiation-induced warping of disks around WDs in symbiotic binaries. Therefore, we first briefly introduce main results of its application to selected types of objects. 

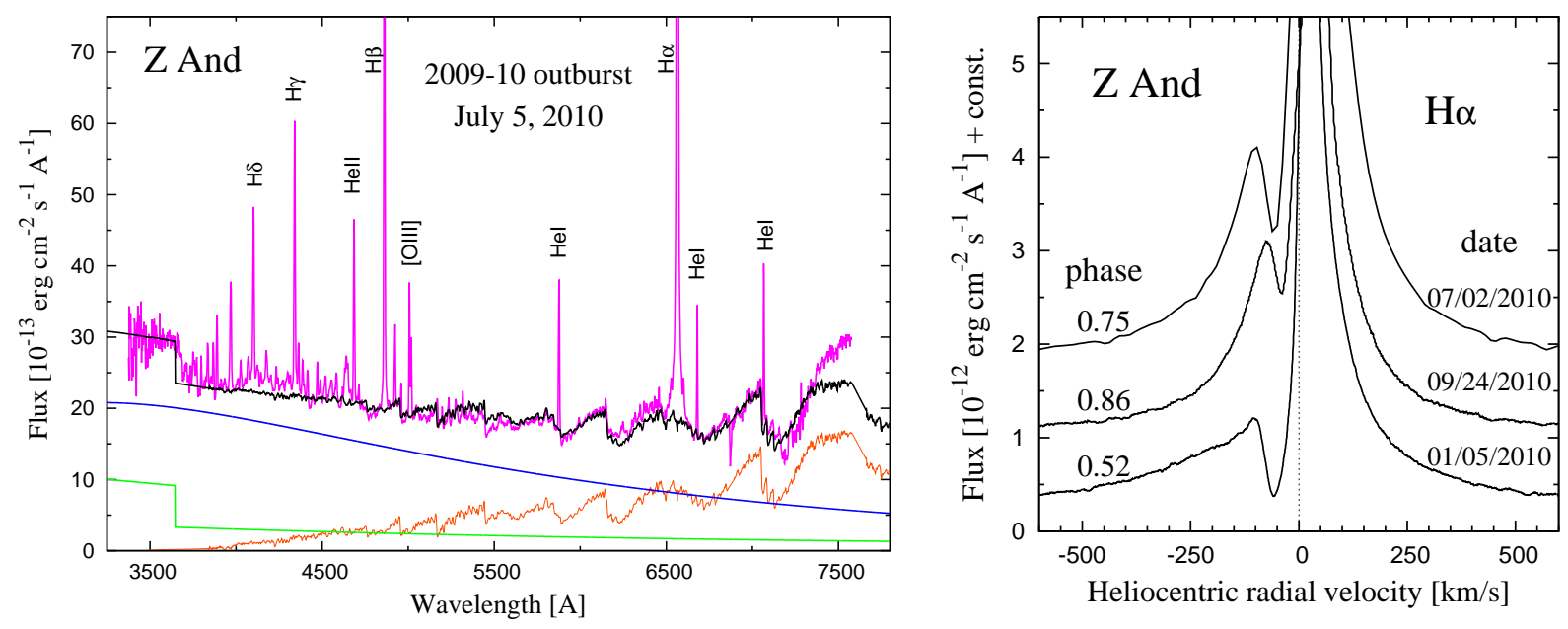

FIG. 5.- Left: Optical spectrum from the 2009-10 outburst (magenta line) and its model SED (solid black line). It is given by superposition of radiation from the warm pseudophotosphere (blue line), the cool giant (orange line) and the nebula (green line). Right: Absorption component in the P-Cyg profile of the $\mathrm{H} \alpha$ line during this outburst indicates expansion of the warm pseudophotosphere.

\subsection{On the radiation-induced warping of disks}

Petterson (1977) found that the effect caused by the pressure of radiation from the center of an $\mathrm{X}$ ray source on the disk structure is very important. Iping \& Petterson (1990) confirmed that radiation forces can make rings of the disk precess in either direction, and change their inclination angle. In this way, they explained the inclination of the disk in Her X-1.4 Using a simple analytic approach, Pringle (1996) showed that even an initially flat disk is unstable to warping, because the surface of a warped disk is illuminated by a central radiation source in a non-uniform manner. Livio \& Pringle (1996) showed that also the accretion disk around the central stars of $\mathrm{PNe}$ can become unstable to a radiationinduced self-warping. Livio \& Pringle (1997) demonstrated that this effect is accompanied by a wobbling motion in both the inclination and azimuthal directions. They used the term "wobble" to describe rather erratic motions. Pringle (1997) investigated the effect of radiation-induced warping on accretion disks around massive black holes in active galactic nuclei. He calculated that the axis of the jets can be severely misaligned from the normal to the outer disk. Southwell et al. (1997) derived radiation-induced precession of the disk with a timescale of the order of months for a strong supersoft X-ray source CAL 83. Wijers \& Pringle (1999) explored the effect of self-induced warping of disks in different types of X-ray binaries. They found that at high luminosities the inner disk can tilt through more than $90^{\circ}$, which may explain the torque reversals in systems such as 4U 1626-675

For symbiotic stars, a disruption of the disk has been considered for the accretion-powered symbiotic system CH Cyg by Sokoloski \& Kenvon (2003). They interpreted the change of the fastest variations (timescale of minutes) into smooth, hour-timescale variations by disruption of the inner disk in association with the mass

\footnotetext{
${ }^{4}$ Her X-1 is an intermediate-mass X-ray binary containing an accreting neutron star with jets (van den Eijnden et al. 2018).

$54 \mathrm{U} 1626-67$ is an ultra-compact X-ray binary bearing a neutron star with the orbital period of $42 \mathrm{~min}$ (Beri et al. 2018, and references therein).
}

ejection event. For the nuclearly powered symbiotic star $\mathrm{Z}$ And, Skopal et al. (2009) suggested that the jets ejection during its 2006 outburst could be also triggered by the radiation-induced disk warping.

In the following section, we will show how the basic conditions for the disk warping and jet ejection are supported by observations for the Z And outbursts with jets.

\subsection{Indication of radiation-induced warping of the disk during $Z$ And outbursts with jets}

According to Sect. 4.1 the necessary ingredients for disk warping and jets ejection are: (i) The presence of a disk, (ii) emergence of a strong central radiation source, and (iii) an observational response of the wobbling motions of the disk. The corresponding critical results confirming these terms for the case of $\mathrm{Z}$ And are described as follows.

(i) The presence of a disk-like formation around the WD during active phases of symbiotic stars was proven by modeling their UV to near-IR SED (see Skopal 2005). The disk-like structure is indicated by the twotemperature-type of the hot component spectrum. The cooler spectrum is produced by a warm stellar pseudophotosphere radiating at $1-2 \times 10^{4} \mathrm{~K}$, whereas the hotter one is represented by the highly ionized emission lines and a strong nebular continuum. The former is not capable of producing the observed nebular emission and thus the latter signals the presence of a strong ionizing source $\left(\gtrsim 10^{5} \mathrm{~K}\right)$ in the system, which is not seen directly by the outer observer. This type of the SED can be explained by a disk-like structure of the hot component viewed under a high inclination angle. Then the flared outer rim of the disk (which is the warm pseudophotosphere indicated by model SED) occults the central ionizing source in the line of sight, while the nebula above/below the disk can easily be ionized (see Fig. 27 of Skopal 2005).

During the 2009-10 outburst of Z And, creation of such a disk around the burning WD was documented by models of SED from September 2009 to November 2010 (see Fig. 8 of Tarasova \& Skopal 2012). Here, Fig. 5] shows example of a representative model SED of the optical spectrum from July 5, 2010. The two- 


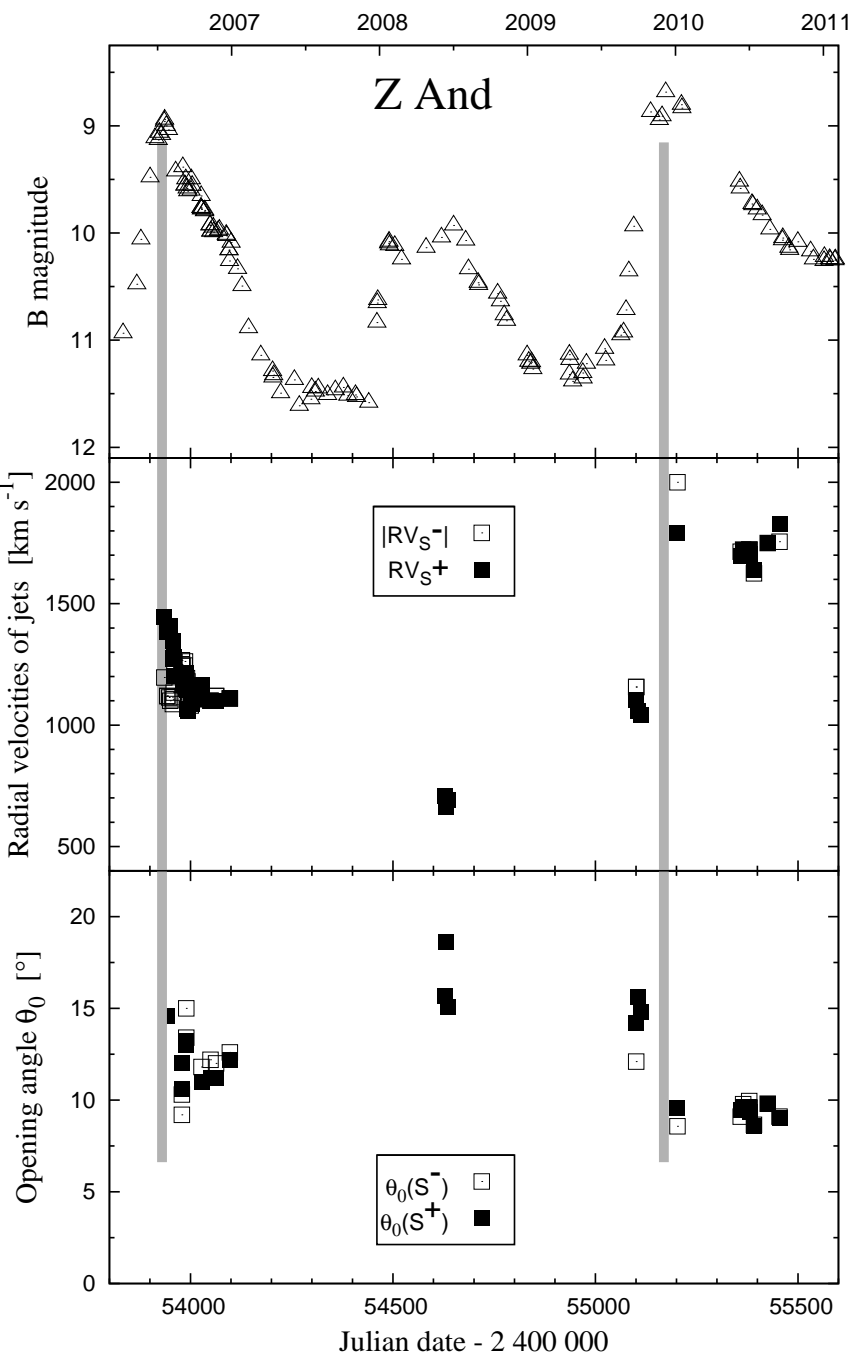

FIG. 6.- Evolution of $R V_{\mathrm{S}}$ (middle) and $\theta_{0}$ (bottom) along the $B$-LC (top) of the 2006, 2008 and 2009-10 outbursts. Maxima of 2006 and 2009-10 events are denoted by vertical bars. Uncertainties in $R V_{\mathrm{S}}$ and magnitudes are within the size of points, and those in $\theta_{0}$ are described in Sect. 3.4.1 Data are from Burmeister \& Leediärv (2007), Tomov et al. (2007), Skopal et al. (2009) and our Table 2

temperature-type of the hot component spectrum consists of a stellar radiation produced by a warm pseudophotosphere (the cooler component) and a strong nebular emission (the hotter component). The former radiates at $\sim 9000 \mathrm{~K}$ and has the luminosity of $\sim 700 L_{\odot}$ (the blue line in the figure), while the latter composes of the nebular continuum with a high emission measure $E M \sim 1.7 \times 10^{60} \mathrm{~cm}^{-3}$ (the green line) and emission lines of highly ionized elements (e.g. H I, He I, He II, [O III]). The warm pseudophotosphere generates the flux of hydrogen ionizing photons $L_{\mathrm{H}} \sim 3 \times 10^{42} \mathrm{~s}^{-1}$, which is not capable of producing the observed $E M$ that requires $L_{\mathrm{H}}=\alpha_{\mathrm{B}}\left(\mathrm{H}, T_{\mathrm{e}}\right) \times E M \sim 2 \times 10^{47} \mathrm{~s}^{-1}$ for the total hydrogen recombination coefficient $\alpha_{\mathrm{B}}\left(\mathrm{H}, T_{\mathrm{e}}\right)=1.0 \times 10^{-13}$ $\mathrm{cm}^{3} \mathrm{~s}^{-1}$ (e.g., Nussbaumer \& Vogel 1987). This implies that the warm pseudophotosphere cannot be a sphere, but a disk, as described above. Independently, the presence of jets indicates the presence of a disk, because jets require an accretion disk (e.g. Livio 1997).

(ii) The emergence of a strong central radiation source is connected with outbursts, when a significant increase of the central source luminosity is indicated. For example, the emission measure of $1.7 \times 10^{60} \mathrm{~cm}^{-3}$ requires the luminosity of the ionizing source to be of the order of $10^{37} \mathrm{erg} \mathrm{s}^{-1}$ (see Skopal et al. 2017, in detail). Also a high $E M \sim 9.4 \times 10^{60} \mathrm{~cm}^{-3}$, as indicated around the maximum of the 2006 outburst (see Sect. 3.1. of Skopal et al. 2009), suggests similarly high luminosity. For the 2000-03 outburst, Sokoloski et al. (2006) estimated the hot star luminosity to $10^{4} L_{\odot}$, lasting approximately for one year. Thus the high luminosity of the central source during outbursts can illuminate the disk, which can become unstable to warping.

(iii) Observational response of the radiation-induced warping of the disk can be associated with the higheramplitude photometric variability $(\Delta m \sim 0.06 \mathrm{mag})$ on the timescale of hours that emerges exclusively during major outbursts, during which the bipolar jets are launched (Fig.4). According to models SED, this type of variability is produced by the warm pseudophotosphere, i.e., the outer rim of the disk, because its contribution dominates the $B, V$ passbands (see Fig. 5 and point (i) above). In this way, we directly indicate the response of the disk warping in the form of the short-term variations in the continuum. As the dynamical timescale of the disk is comparable with the timescale of the smooth light variations (see Appendix $\underline{\mathrm{A}}$ ), this can be caused by a variable projection of the disk surface into the line of sight. We thus observe wobbling of the outer parts of the disk - in agreement with the general view that the warped disk starts to wobble or precess (Livio \& Pringle 1997, Sect. 4.1). Alternatively, the smooth light variations could be partly caused by the brightness variation of the outer disk due to reprocessing flickering light from its inner warping part (see Appendix B).

\subsection{Jets' radial velocities from the warping disk}

Complex variations in $R V_{\mathrm{S}}$ around the maxima of both 2006 and 2009-10 outbursts (Sect. 3.5) could also be a direct result of the radiation-induced disk warping. Assuming jets to be perpendicular to the inner disk, their initial chaotic wobbling motions can produce shifts in $R V_{\mathrm{S}}$ due to the different inclination of a jet to the line of sight, as considered for PNe by Livio \& Pringle (1997). One can also imagine that the twisted inner disk can cause that axis of both jet nozzles not to be parallel, which causes the observed jet's asymmetry (see Fig. 6 of Skopal et al. 2009).

However, after a short time of $\sim 1$ and a few months, the values of $R V_{\mathrm{S}}$ settled at around $\pm 1100 \mathrm{~km} \mathrm{~s}^{-1}$ and $\pm(1700-1800) \mathrm{km} \mathrm{s}^{-1}$ for 2006 and $2009-10$ outburst, respectively. During the small 2008 burst, the only present $\mathrm{S}^{+}$component was located at $\sim 700 \mathrm{~km} \mathrm{~s}^{-1}$ (Table 2). This suggests that the wobbling motions of the disk ceased.

Diversity of the final $R V_{\mathrm{S}}$ during each outburst could be caused by different conditions for the radiation-driven warping during these events. According to Pringle (1996), the radiation-driven warping of the disk can occur at radii, which depend on the central star mass and luminosity, accretion rate, and viscosities in the disk. During the Z And-type outbursts, additional energy is liberated by thermonuclear burning on the WD 
surface due to a transient high accretion rate (see, e.g., Skopal et al. 2017, for AG Peg). Depending on its quantity during different outbursts with different properties of the disk, the warping can occur at different radii. Qualitatively, the material liberated at different heights above the WD then converts different amounts of the gravitational potential energy into the kinetic energy at its accretion and thus can be expelled at different velocities in the form of jets. The energy power of these outbursts, as given by their brightness maximum and duration, seems to be consistent with this view (see Fig. 1).

\subsection{Disk-jets connection in $Z$ And}

Figure 7 shows the evolution of the measured flux of jets and the effective radius of the warm pseudophotosphere, $R_{\mathrm{WD}}^{\mathrm{eff}}$, along the 2006 and 2009-10 outbursts. Note that the disk radius $R_{\mathrm{D}} \propto R_{\mathrm{WD}}^{\mathrm{eff}}$ (e.g., Eq. (11) of Skopal et al. 2011). Effective radii were derived from modeling the SED during the 2009-10 outburst (see Table 5 of Tarasova \& Skopal 2012). From the 2006 outburst, there is only one estimate of $R_{\mathrm{WD}}^{\mathrm{eff}}$ available around the optical maximum (Skopal et al. 2009). Gradual decrease of the disk radius and the flux of jets along the decline of the star's brightness implies a gradual dilution of the disk.

Shrinking of the disk radius is also indicated by the radial velocities of the absorption component in the $\mathrm{H} \alpha$ profile that decelerate along the decline of the star's brightness (bottom panel of Fig. 7). This effect can be a result of expansion of the outer parts of the disk leading to a shrinkage of the optically thick part of the warm pseudophotosphere. Therefore, the optically thick wind in the $\mathrm{H} \alpha$ line at/above the warm pseudophotosphere will also originate at smaller radii, where it is driven outside with a smaller velocity.

Below we describe how the disk-jets connection can be understood in the context of the accretion process onto the WD during $\mathrm{Z}$ And outbursts with jets.

\subsubsection{On the accretion during $Z$ And outbursts with jets}

Significant increase of the WD luminosity around the optical maximum, the indication of the disk warping (Sects. 4.2 and 4.3), and the presence of jets is a result of a significant increase of the accretion rate through the disk, so that an excess of angular momentum of the accreting material has to be removed via jets. A fraction of the disk material, accreted onto the WD, enhances nuclear shell burning on its surface. As a consequence, the high radiation output makes the surrounding disk unstable to warping and enhances the stellar wind during outbursts (see Sect.4.5 for some details). The rest of the accreting material is liberated in the form of jets, whose kinetic energy comes from their accretion energy. This leads to a gradual removal of the disk material from its inner parts onto the WD and into the jets. As a result, the gradual decrease of fueling the WD and the release of the accretion energy lead to a gradual decline of the star's brightness, shrinking of the disk radius and vanishing of the jets as documented by observations in Fig. 7.

In a nutshell, expanding of the outer parts of the disk and sucking its inner parts by the luminous WD via the radiation-induced warping lead to a gradual dilution of the disk. This gives reasons for the observed disk-jets

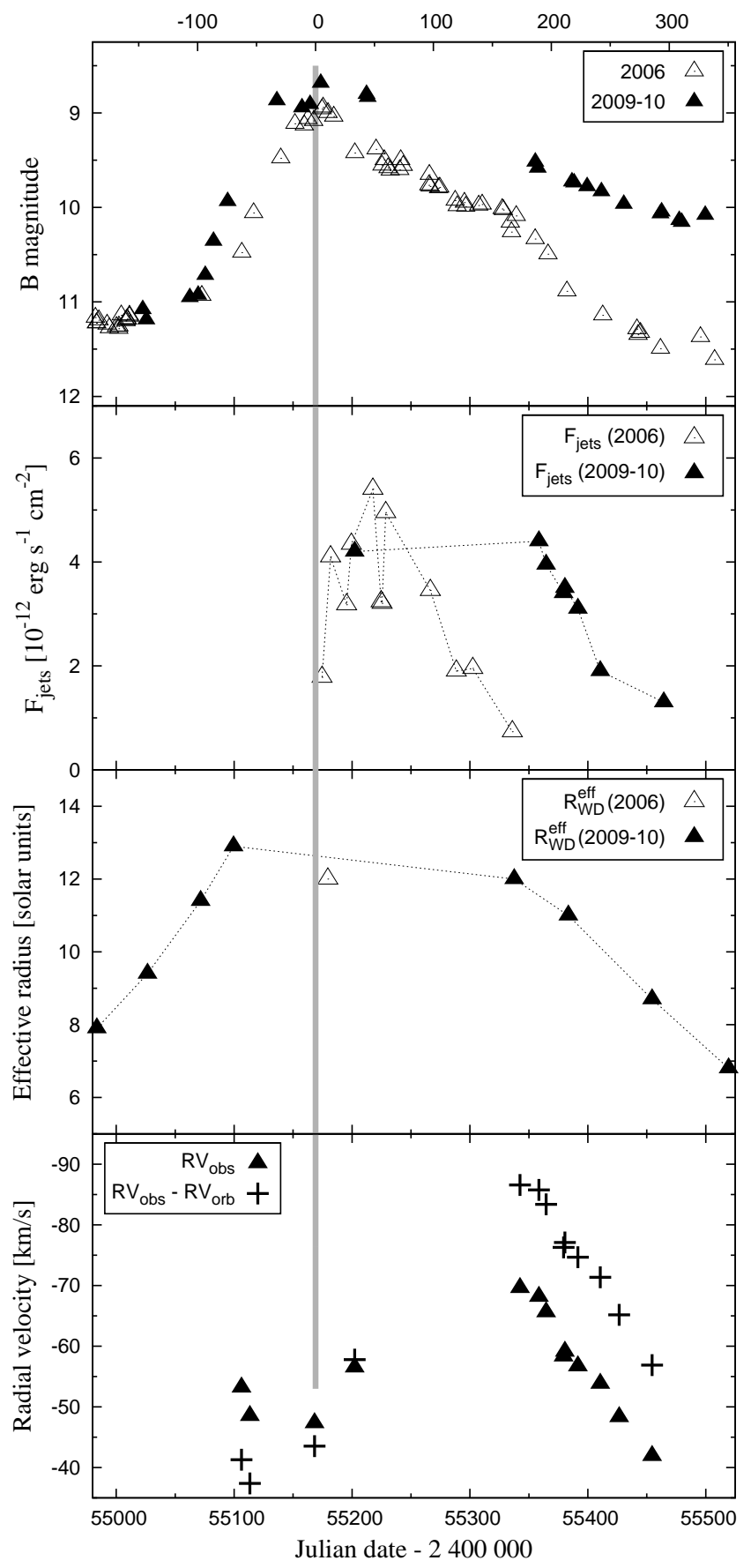

FIG. 7.- Disk-jets connection during the 2006 and 2009-10 outbursts. The top panel compares their $B$-LCs shifted to their maxima (vertical bar). The second panel shows evolution of average fluxes of jets, $F_{\text {jets }}=\left(F_{\mathrm{S}}++F_{\mathrm{S}^{-}}\right) / 2$ (data from Tomov et al. 2007; Skopal et al. 2009, and our Table 2). The third panel plots the evolution of the effective radius, $R_{\mathrm{WD}}^{\mathrm{eff}}$, of the warm pseudophotosphere, and the bottom panel displays radial velocities of the absorption component in the $\mathrm{H} \alpha$ profile (crosses are values corrected for the orbital motion of the hot component according to elements of Fekel et al. (2000)). 
connection and explains why the launching of jets is transient.

\subsection{On jets and $Z$ And-type outbursts}

During quiescent phases of symbiotic stars, no jets have been indicated, although their WDs accrete throughout the accretion disk and, in most cases, at high rates of $10^{-8}-10^{-7} M_{\odot} \mathrm{yr}^{-1}$ to power their high luminosities by stable hydrogen burning on the WD surface (e.g., Paczyński \& Zytkow 1978; Hachisu et al. 1996; Shen \& Bildsten 2007). To date, jets were indicated only during outbursts (see Sect. 1), during which a large optically thick disk is created around the WD (Skopal 2005). The presence of jets thus confirms the presence of the disk and constrains accretion at a high rate, although enhanced mass-outflow from the hot component is directly indicated (see below).

For the nuclearly powered symbiotics, the appearance of transient jets signals transient accretion at rates above the limit of the stable hydrogen burning on the WD surface. According to theoretical predictions, at these rates the luminosity of the burning WD can increase to the Eddington limit (e.g., Fig. 2 of Shen \& Bildsten 2007), and the mass-outflow from the WD in the form of wind enhances (Hachisu et al. 1996). Both characteristics are well supported by observations. (i) The high luminosity of the WD, burning hydrogen above the stable limit, was recently evidenced for AG Peg during its 2015 Z Andtype outburst (Skopal et al.|2017). For Z And, the WD's luminosity of the order of $\sim 10^{37} \mathrm{erg} \mathrm{s}^{-1}$ was determined for the 2000-03, 2006 and 2009-10 outbursts (see the point (ii) of Sect. 4.2). (ii) Enhanced mass-outflow via the wind is directly indicated by broadening of emission lines during outbursts (e.g. Fernández-Castro et al. 1995). Modeling the broad H $\alpha$ wings, Skopal (2006) found an increase of the mass-loss rate via the wind from hot components during outbursts to $\gtrsim 10^{-6} M_{\odot} \mathrm{yr}^{-1}$, i.e., factor of $\gtrsim 10$ higher with respect to values from quiescent phases.

Appearance of jets during Z And-type outbursts thus manifests their nature by nuclear burning of hydrogen on the WD surface at rates above the upper limit of the stable burning. The highly energetic events we observe during these outbursts (the luminosity close to the Eddington value and the wind outflow at $\gtrsim 10^{-6} M_{\odot} \mathrm{yr}^{-1}$ ) require a relevant source of material that effectively fuels the WD. Indication of a large neutral disk at/around the orbital plane during Z And-type outbursts was described in the point (i) of Sect. 4.2. According to Cariková \& Skopal (2012) such a disk can be formed during outbursts by compression of the enhanced wind toward the equatorial plane due to rotation of the WD. This means that the disk consists of the material previously accreted onto the WD from the giant. Due to the thermal warping, this material is reaccreted again, which prolongs the period with a high luminosity. The continuous flow of material from the giant's wind helps to refill in the disk until the next possible instability will cause another (out)burst. This process can repeat up to depletion of the disk formed during outbursts. Then an accretion disk will be created from the giant's wind, and the system enter a quiescent phase.

According to Leibowitz \& Formiggini (2008), the main active phases of Z And last approximately of 15-20 years and appear quasi-periodically with a separation of their centers by $\approx 20$ years (see their Fig. 1 ). Assuming this evolution also for the current active phase, it should cease around 2020.

\section{SUMMARY}

We continued monitoring of the prototypical symbiotic star Z And after its major 2006 outburst, during which two-sided jets in the optical spectrum were indicated for the first time. We used the high-resolution spectroscopy around $\mathrm{H} \alpha$, multicolor $U B V R_{\mathrm{C}}$ photometry, and hightime-resolution photometry to search for the reappearance of jets during the following outbursts of the current active phase (Fig. 1). Our findings may be summarized as follows.

1. The bipolar jets reappeared during the major 200910 outburst, as indicated by well-pronounced $\mathrm{S}^{-}$ and $\mathrm{S}^{+}$satellite components to the $\mathrm{H} \alpha$ line. During the smaller 2008 burst, only a single $\mathrm{S}^{+}$component was present (Sect. 3.2. Figs. 2 and 3).

2. The evolution of jets during both the 2006 and the 2009-10 outbursts was similar. The jets appeared around the outburst maxima, weakened along the decline of the star's brightness, and ceased after $\approx 5$ and $\approx 10$ months during 2006 and 2009-10 outbursts. A large variation in jets radial velocities was measured at their emergence, but after 13 months they settled on a constant level of \pm 1100 and $\sim \pm 1750 \mathrm{~km} \mathrm{~s}^{-1}$, respectively (Sect.3.5 Fig. 6).

3. During both outbursts, a smooth light variation within $\Delta B \sim 0.06 \mathrm{mag}$ on the timescale of hours developed from rapid, $\lesssim 0.02 \mathrm{mag}$, stochastic fluctuations outside the outbursts (Sects. 3.1 and 3.5. Fig. 4). This type of variability is produced by the outer rim of the flared disk that develops during outbursts (see point (iii) of Sect.4.2).

4. Ejection of jets with simultaneous emergence of the hours-timescale photometric variation and the measured disk-jets connection can be caused by the radiation-induced warping of the inner disk due to the outburst of the central WD (Sects. 4.2, 4.3 and 4.4).

5. Jets launched by nuclearly powered symbiotics require transient accretion at rates above the upper limit of the stable hydrogen burning on the WD surface. The jets thus prove the nature of $\mathrm{Z}$ And-type outbursts by their ignition at these rates (Sect.4.5). Then the WD generates the luminosity of the order of $10^{4} L_{\odot}$ and drives the wind at $\gtrsim 10^{-6} M_{\odot} \mathrm{yr}^{-1}$.

6. The large disk created at the beginning of the outbursts $\left(R_{\mathrm{D}} \sim 10-20 R_{\odot}\right.$, Fig. 7) consists of the material originally accreted onto the WD from the giant (Cariková \& Skopal 2012). It represents a reservoir of mass for fueling the burning WD, because its inner part can be reaccreted via the radiation-induced warping. In this way, a high luminosity of the burning WD can be sustained for a 
longer time, until depletion of the disk (Sect. 4.5). The jets thus signal the presence of the reaccretion process, providing a link to long-lasting active phases of Z And.

Observations of transient jets along the decline from the optical maximum of some $\mathrm{Z}$ And-type outbursts (to-date observed for Hen 3-1341 (Munari et al. 2005), Z And (e.g. Burmeister \& Leediärv 2007) and BF Cyg (Skopal et al. 2013)) represent new challenges for the theoretical modeling of the radiation-induced warping of disks formed during outbursts of symbiotic stars. This should provide us with a better understanding of the accretion process during nuclearly powered eruptions on the surface of WDs with prolonged stages of high luminosity and signatures of collimated mass ejection.

The authors thank the anonymous referee for critical, but encouraging, comments on the original version of the manuscript. Theodor Pribulla is thanked for acquisition of spectra at the David Dunlap Observatory. Miloslav Tlamicha, Tereza Krejčová, Pavel Chadima, Lenka Kotková, Lukáč Pilarčík, Jan Sloup, Kateřina Hoňková, Jakub Juryšek, Luděk Rezba and Jan Fuchs, are thanked for their assistance in the acquisition of the spectra at the Ondřejov observatory. We also acknowledge the variable-star observations from the AAVSO International Database contributed by observers worldwide and used in this research. This work was supported by the Czech Science Foundation, grants P209/10/0715 and GA15-02112S, by the Slovak Research and Development Agency under the contract No. APVV-15-0458, by the Slovak Academy of Sciences grant VEGA No. $2 / 0008 / 17$ and by the realization of the project ITMS No. 26220120029, based on the supporting operational Research and development program financed from the European Regional Development Fund.

\section{APPENDIX}

\section{DYNAMICAL TIMESCALE OF THE DISK}

Here we compare the timescale of the observed light variability (Sect. 3.1) with the dynamical time of the disk with radius $R_{\mathrm{D}}$ around the WD with the mass of $M_{\mathrm{WD}}$,

$$
t_{\mathrm{dyn}}=0.44 \times\left(\frac{R_{\mathrm{D}}}{R_{\odot}}\right)^{2 / 3}\left(\frac{M_{\mathrm{WD}}}{M_{\odot}}\right)^{-1 / 2} \mathrm{hr} .
$$

A representative value of $R_{\mathrm{D}}=10 R_{\odot}$ (see Fig. 7) and $M_{\mathrm{WD}}=1 M_{\odot}$ corresponds to $t_{\mathrm{dyn}} \sim 14$ hrs. If we consider $R_{\mathrm{D}} \sim 5 R_{\odot}$ for the outer part of the disk, then $t_{\mathrm{dyn}} \sim 5 \mathrm{hrs}-$ well comparable with the variation in the optical light as measured during the outbursts with jets. On the other hand, shorter variations from a low state, $\lesssim 1 \mathrm{hr}$ (Fig. 4 ), correspond to $R_{\mathrm{D}} \lesssim 1.5 R_{\odot}$.

\section{DIFFUSION TIMESCALE OF THE DISK}

A contribution to the smooth light variation could also come from the inner warping part of the disk, whose flickering light is hidden, but can be reprocessed in the outer disk. The timescale for this process is given by the diffusion timescale,

$$
t_{\mathrm{dif}}=R_{\mathrm{out}}^{2} / c l,
$$

where $R_{\text {out }}$ is the radius of the reprocessing medium, $c$ is the light speed, and $l$ is the mean-free path of a photon. For the opacity $\kappa_{\lambda}$ and density $\rho$ of the medium, $l=1 / \kappa_{\lambda} \rho$. If we can assume that $\rho$ and $\kappa_{\lambda}$ are constant along the path of the photon, then the corresponding optical depth $\tau_{\lambda}=\kappa_{\lambda} \rho R_{\text {out }}=R_{\text {out }} / l$, and the diffusion timescale can be expressed as $R_{\text {out }} \tau_{\lambda} / c$, or

$$
t_{\text {dif }}=6.45 \times 10^{-4} \frac{R_{\text {out }}}{R_{\odot}} \tau_{\lambda} \mathrm{hr} .
$$

If the thickness $R_{\text {out }} \sim 5 R_{\odot}$ and its total optical depth is as large as $1-10^{4}$, the diffusion timescale $t_{\text {dif }}=12 \mathrm{~s}-1.3$ days is sufficiently short to observe the inner disk light variation at its outer rim (which is the warm pseudophotosphere), well within the timescale of the jet ejection period ( $\approx 1$ years).

\section{REFERENCES}

Beri, A., Paul, B., \& Dewangan, G. C. 2018, MNRAS, 475, 999

Bessel, M. S. 1979, PASP, 91, 589

Boyarchuk, A. A. 1967, Soviet Astronomy, 11, 8

Burmeister, M., \& Leedjärv, L. 2007, A\&A, 461, L5

Brandi, E., Mikołajewska, J., Quiroga, C. et al. 2005, A\&A, 440, 239

Cariková, Z., \& Skopal, A. 2012, A\&A, 548, A21

Esipov, V. F., Kolotilov, E. A., Mikołajewska, J., et al. 2000, Astronomy Letters, 26, 162

Fekel, C. F., Hinkle, K. H., Joyce, R., \& Skrutskie, M. F. 2000, AJ, 120, 3255

Fernández-Castro, T., González-Riestra, R., Cassatella, A., Taylor, A. R., \& Seaquist E. R. 1995, ApJ, 442, 366
Fluks, M. A., Plez, B., The, P. S., de Winter, D., Westerlund, B. E., \& Steenman, H. C. 1994, A\&AS, 105, 311

Hachisu, I., Kato, M., \& Nomoto, K. 1996, ApJ, 470, L97

Henden, A. A., \& Kaitchuck, R. H. 1982, Astronomical Photometry, (New York: Van Nostrand Reinhold Company), 50 Henden, A. A., \& Honeycutt, R. K. 1997, PASP, 109, 441 Horn, J., Kubát, J., Harmanec, P., et al. 1996, A\&A, 309, 521 Iping, R. C., \& Petterson, J. A. 1990, A\&A, 239, 221

Kenyon, S. J. 1986, The symbiotic stars, CUP, New York Leibowitz, E. M., \& Formiggini, L. 2008, MNRAS, 385, 445 Leibowitz, E. M., \& Formiggini, L. 2013, ApJ, 146:117

Livio, M. 1997, in ASP Conf. Ser. 121, Accretion Phenomena and Related Outflows, ed. D. T. Wickramashinge, G. V. Bicknell, \& L. Ferrario, (San Francisco: ASP), 845 
Livio, M., Pringle, J. E. 1996, ApJ, 465, L55

Livio, M., Pringle, J. E. 1997, ApJ, 486, 835

McKeever, J., Lutz, J., Wallerstein, G., Munari, U., \& Siviero, A. 2011, PASP, 123, 1062

Mikołajewska J., \& Kenyon, S. J. 1996, AJ, 112, 1659

Munari, U., Tomov, T., Yudin, B. F., et al. 2001, A\&A, 369, L1

Munari, U., Siviero, A., \& Henden, A. 2005, MNRAS, 360, 1257

Mürset, U., Nussbaumer, H., Schmid, H. M., \& Vogel, M. 1991, A\&A, 248, 458

Mürset, U., \& Schmid, H. M. 1999, A\&AS, 137, 473

Nussbaumer, H., \& Vogel, M. 1987, A\&A, 182, 51

Nussbaumer, H., \& Vogel, M. 1989, A\&A, 213, 137

Osterbrock, D. E. 1989, Astrophysics of Gaseous Nebulae and Active Galactic Nuclei, (San Francisco: W. H. Freema and Company Press)

Pacźynski, B., \& Żytkow, A. N. 1978, ApJ, 222, 604

Paczyński, B., \& Rudak, R. 1980, A\&A, 82, 349

Parimucha, Š., \& Vaňko, M. 2005, CoSka, 35, 35

Petterson, J. A. 1977, ApJ, 216, 827

Pringle, J. E. 1996, MNRAS, 281, 357

Pringle, J. E. 1997, MNRAS, 292, 136

Seaquist, E. R., Taylor, A. R. \& Button, S. 1984, ApJ, 284, 202

Shagatova, N. 2017, PASP, 129, 067001

Shen, K. J., \& Bildsten, L. 2007, ApJ, 660, 1444

Škoda, P. 1996, in ASP Conf. Ser. 101, Astronomical Data Analysis Software and Systems V, ed. G. H. Jacoby, \& J.

Barnes, (San Francisco: ASP), 187

Skopal, A. 2005, A\&A, 440, 995

Skopal, A. 2006, A\&A, 457, 1003

Skopal, A. 2007, New Astron., 12, 597

Skopal, A., Chochol, D., Pribulla, T., Vaňko, M. 2000, IBVS No. 5005
Skopal, A., \& Pribulla, T. 2006, ATel 882

Skopal, A., Vittone, A. A., Errico, L., et al. 2006, A\&A, 453, 279

Skopal, A., Pribulla, T., Budaj, J., et al. 2009, ApJ, 690, 1222

Skopal, A., Tarasova, T. N., Cariková, Z., et al. 2011, A\&A, 536, A27

Skopal, A., Shugarov, S., Vaňko, M., et al. 2012, AN, 333, 242

Skopal, A., Tomov, N. A. \& Tomova, M. T. 2013, A\&A, 551, id L10

Skopal, A., Shugarov, S. Yu., Sekeráš, M., et al. 2017, A\&A, 604, A48

Sokoloski, J. L., \& Kenyon, S. J. 2003, ApJ, 584, 1021

Sokoloski, J. L., Kenyon, S. J., Espey, B. R., et al. 2006, ApJ, 636, 1002

Southwell, K. A., Livio, M., \& Pringle, J. E. 1997, ApJ, 478, L29

Tarasova, T. N., \& Skopal, A. 2012, ARep, 56, 212

Tomov, T., Kolev, D., Zamanov, R., Georgiev, L., \& Antov, A. 1990, Nature, 346, 637

Tomov, T., Munari, U., \& Marrese, P. M. 2000, A\&A, 354, L25

Tomov, N. A., Tomova, M. T., \& Bisikalo, D. V. 2007, MNRAS, 376, L16

Tomov, T., Zamanov, R., Gałan, C., \& Pietrukowicz, P. 2017, AcA, 67, 225

van den Eijnden, J., Degenaar, N., Russell, T. D., et al. 2018, MNRAS, 473, L141

Vaňko, M., Komžík, R., Kollár, V., \& Sekeráš, M. 2015a, CoSka, 44, 77

Vaňko, M., Komžík, R., Kollár, V., \& Sekeráš, M. 2015b, CoSka, 44, 91

Wijers, R. A. M. J. \& Pringle, J. E. 1999, MNRAS, 308, 207 